\title{
Desenvolvimento vegetativo e características físico-químicas dos frutos de quatorze genótipos de limão
}

\author{
Rodrigo do Vale Ferreira ${ }^{1 *}$, Marinês Bastianel ${ }^{2}$, Fernando Alves de Azevedo ${ }^{2}$ \\ \& José Dagoberto De Negri ${ }^{2}$
}

\begin{abstract}
RESUMO
O limão, também conhecido como limão verdadeiro [Citrus limon (L.) Burm. f.] ainda tem sua origem controvertida, mas a hipótese mais considerada é que seja um híbrido originário da Índia. Essa fruta tem grande importância no mundo, sendo que as principais regiões de cultivo são os Estados Unidos da América (EUA), Argentina e região mediterrânea. No Brasil sua importância ainda é restrita à fabricação de refrigerantes e em menor quantidade ao mercado in natura. As principais cultivares no país são os limões Siciliano e Eureka. O objetivo desse trabalho foi avaliar o desenvolvimento da copa e a qualidade físico-química dos frutos de quatorze genótipos de limão do Banco Ativo de Germoplasma de Citros (BAG Citros), do Centro de Citricultura Sylvio Moreira/IAC, localizado em Cordeirópolis, SP. Os genótipos foram mensurados quanto a altura e o diâmetro para a obtenção do volume de copa e determinação do seu desenvolvimento. Os frutos foram submetidos à análise de determinação de seus parâmetros físicos e químicos e a consequente distinção entre eles. Os resultados mostraram grande variação nos materiais estudados quanto ao desenvolvimento da copa, sendo que os cultivares Allen Eureka e Genova apresentaram menor porte, com potencial para plantios adensados. Quanto à qualidade de fruto os genótipos Meyer e Genova se destacaram com maiores teores de sólidos solúveis, vitamina $\mathrm{C}$ e rendimento em suco.
\end{abstract}

Termos de indexação: Citrus limon, Siciliano, qualidade.

\section{Vegetative development and physicochemical characteristics of the fruits of fourteen lemon genotypes}

\section{SUMMARY}

The lemon, also known as true lemon, [Citrus limon (L.) Burm. f.] still has its origin in controversy, but the hypothesis most considered is that it is a hybrid originating in India. This fruit is of great importance in the world, with the main growing regions being the United States of America (USA), Argentina and the Mediterranean region. In Brazil its importance is still restricted to the manufacture of soft drinks and in smaller quantity to the market in natura. The main cultivars in the

\footnotetext{
${ }^{1}$ Programa de Desenvolvimento Tecnológico e Industrial - DTI, Conselho Nacional de Desenvolvimento Científico e Tecnológico - CNPq, Centro de Citricultura Sylvio Moreira, Insituto Agranômico - IAC, Agência Paulista de Tecnologia dos Agronegócios APTA, Cordeirópolis, SP, Brasil

${ }^{2}$ Centro de Citricultura Sylvio Moreira, Insituto Agranômico - IAC, Agência Paulista de Tecnologia dos Agronegócios - APTA, Cordeirópolis, SP, Brasil

*Autor correspondente: Rodrigo do Vale Ferreira, Centro de Citricultura Sylvio Moreira, Insituto Agranômico - IAC, Agência Paulista de Tecnologia dos Agronegócios - APTA, Rodovia Anhanguera, Cascalho, km 158, CP 4, CEP 13490-970, Cordeirópolis, SP, Brasil. E-mail: rodrigodovaleferreira@yahoo.com.br
} 
country are Sicilian and Eureka lemons. The objective of this work was to evaluate the development of the canopy and the physicochemical quality of the fruits of fourteen lemon genotypes of the germplasm collection (BAG Citros), from the Centro de Citricultura Sylvio Moreira/IAC, located in Cordeirópolis, Sao Paulo State, Brazil. The materials were measured for height and diameter to obtain canopy volume and determination of their development. The fruits were submitted to analysis of determination of their physical and chemical parameters and the consequent distinction between them. The results showed a great variation in the studied materials, with the cultivars Allen Eureka and Genova presenting smaller size, with potential for densified plantings. As for fruit quality, the Meyer and Genova genotypes showed higher levels of soluble solids, vitamin $\mathrm{C}$ and yield in juice.

Index terms: Citrus limon, Sicilian, quality.

\section{INTRODUÇÃO}

A origem e taxonomia do limão ainda são bastante controversas, mas acredita-se que seu centro de origem seja a Índia. Sua entrada na Europa ocorreu no século XI através das Cruzadas e sua introdução no Continente Americano ocorre com as grandes navegações, principalmente com a chegada de Cristóvão de Colombo às Américas em 1492 (Pio et al., 2005). Curk et al. (2016) em estudo de similaridade filogenética com limas e limões, observaram Citrus medica como sendo componente genômico comum, atuando sempre como doador de pólen em combinação com outras espécies do gênero Citrus.

O limoeiro é uma planta com hábito de crescimento ereto e bastante vigoroso, suas folhas são de formato oval e coloração verde clara, os ramos apresentam espinhos de diferentes tamanhos e as flores e brotações apresentam coloração roxo purpúreo. $\mathrm{O}$ fruto, em comparação à laranja, é mais alongado terminando com duas extremidades proeminentes, é de cor amarela, casca grossa e abundante e levemente rugosa (Alves, 1986). As principais variedades de limões verdadeiros cultivados são: a) Siciliano: é a cultivar mais plantada do Brasil e Estados Unidos, apresenta frutos de tamanho mediano, pouca semente e alto teor de suco, bastante apreciado para mercado de fruta fresca; $b$ ) Femminello: o mais cultivado na Europa, apresenta porte alto e vigoroso, os frutos tem tamanho médio, casca grossa e menor teor de suco; c) Lisboa: apresenta plantas de porte média a alto, bastante cultivado na Argentina e Estados Unidos, muito produtivo, resistente ao frio e apresenta a casca rugosa em relação ao Eureka; d) Genova: é de origem italiana, tem boa resistência ao frio, porte menor, fruto mais arredondado e as qualidades químicas muito semelhante ao Eureka (Pio et al., 2005).

O limão tem diferentes destinos e aproveitamentos, a priori, os frutos de melhor qualidade são utilizados para o consumo no mercado de frutas frescas e os que não se enquadram nessas características são destinados para a produção de suco concentrado, óleos essenciais e pectina (Landa, 2014). É difícil a análise de números sobre a produção e comercialização de limão, visto que são considerados "limões" também as limas ácidas Tahiti (Citrus latifolia (Yu. Tanaka) Tanaka) e Galego (Citrus aurantifolia (Christm) Swingle), mas estima-se que no mundo $70 \%$ da produção seja de limão e o restante de limas ácidas. A produção brasileira, ao contrário, apresenta grande porcentagem de limas ácidas e em menor número limão (Amaro et al., 2003).

Boa parte do limão produzido no Brasil é consumido no mercado interno brasileiro para indústria de refrigerante e uma pequena parte para o consumo in natura (Amaro \& Maia, 1997). A produção mundial de limas e limões é estimada em 16,2 milhões de toneladas em 1 milhão de ha, sendo a Índia, México, China, Argentina e Brasil os principais produtores. No Brasil a produção é de aproximadamente 1,2 milhões toneladas com uma área de 47,2 mil ha (FAO, 2016), destinada para industrialização e mercado de fruta fresca, mas o interesse dos produtores pelo mercado de frutas fresca para o limão verdadeiro, vem aumentando e apresentando um grande potencial, visto que o consumo ainda é pequeno comparado a outros citros.

Para uma boa comercialização de frutas in natura faz-se de extrema importância a qualidade físico-química do material. Esses requisitos englobam coloração da casca e aspectos externos, tamanho apropriado, espessura de casca, suco com adequado equilíbrio da acidez e sólidos solúveis, aroma, pequeno número de sementes, boa conservação e resistência ao transporte (Pio, 1992). Mesmo para frutos destinados ao processamento industrial, onde a qualidade externa do fruto não é tão considerada, as qualidades organolépticas e o valor nutritivo tem grande importância (Carvalho \& Nogueira, 1979).

Dentro do sistema produtivo de citros as práticas agrícolas exercem influência tanto na qualidade do fruto como na produtividade. Nesse contexto, o adensamento de plantio na citricultura é uma tecnologia adotada com objetivo de incrementar a produção por unidade de área e manter produções longevas, mesmo com a perda de plantas por doenças como a gomose, clorose variegada dos citros e, principalmente, o huanglongbing - HLB 


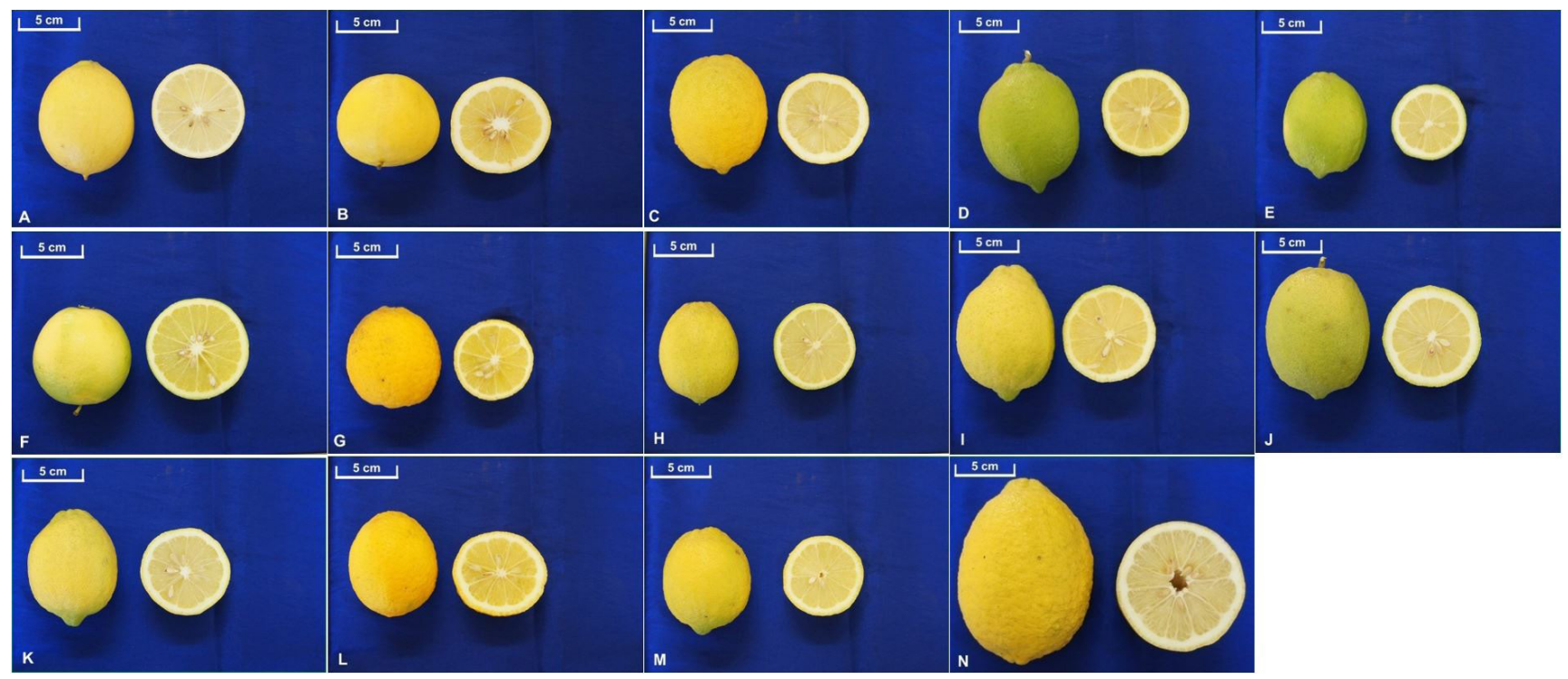

Figura 1. Genótipos de limão selecionados do Banco Ativo de Germoplasma de Citros do Centro de Citricultura Sylvio Moreira/IAC. Cordeirópolis, SP (2016-2017). A- Wolglum; B- São Matheus; C- Rigoni; D- Meyer; E- Lisboa Bocaiuva; F- Jaraguá do Sul; G- Harvey; H- Geórgia; I- Genova; J- Deba Ahmed; K- Cook Eureka; L- Amber; M- Allem Eureka; N- Kusner.

(ex-greening) (Stuchi \& Girardi, 2011). Outra característica desejada em pomares atuais são plantas de menor porte e volume de copa. Essas características, além de facilitar o adensamento, facilita a colheita e tornam mais eficientes e econômicas algumas práticas fitossanitárias. $\mathrm{O}$ adensamento é conseguindo diminuindo-se os espaços entre as linhas de plantio e, principalmente, entre as plantas, essa diminuição não apresenta grande impacto na qualidade dos frutos. Já o porte menor da planta é buscado através do uso de portaenxertos ananicantes e/ou variedades de copa com essa característica, o que influenciam diretamente a qualidade do produto (Donadio \& Stuchi, 2001).

Nesse sentido o objetivo do presente trabalho foi avaliar o desenvolvimento vegetativo da copa e a qualidade físicoquímica dos frutos na época de maturação de quatorze genótipos de limão.

\section{MATERIAL E MÉTODOS}

\section{Material vegetal}

Foram avaliados quatorze genótipos de limão (Citrus limon) enxertados em limoeiro Cravo (C. limonia Osbeck), pertencentes ao Banco Ativo de Germoplasma de Citros (BAG-Citros) do Centro de Citricultura Sylvio Moreira/ IAC que foram plantados em 2015 na coleção de trabalho localizada em Cordeirópolis, SP $\left(22^{\circ} 27^{\prime} 33^{\prime \prime} \mathrm{S} ; 47^{\circ} 24^{\prime}\right.$
17" O). O plantio foi realizado em delineamento de blocos casualizados com quatro repetições e duas plantas por parcela. Os genótipos estudados estão ilustrados na Figura 1 .

\section{Avaliações}

\section{Desenvolvimento vegetativo}

Foram mensurados a altura e diâmetro das plantas com auxílio de uma régua graduada, onde se mediu a planta do colo até o ápice (altura) e na linha horizontal a parte mediana da copa (diâmetro). Determinou-se ainda, o volume da copa pela equação proposta por Mendel (1956): $V=2 / 3 \pi R^{2} H$, onde $V$ representa o volume $\left(\mathrm{m}^{3}\right)$, $\mathrm{R}$, o raio da copa (m) e H, a altura da planta (m). Essas medidas foram realizadas em dois períodos, aos $12 \mathrm{e}$ aos 24 meses após o plantio.

\section{Características físico-químicas dos frutos}

Foram realizadas avaliações físico-químicas dos frutos em seu período de maturação. A coleta dos frutos da florada principal foi realizada em maio de 2017, com um total de cinco unidades em cada planta, por repetição. A colheita foi realizada na porção externa da copa, na faixa compreendida entre 1,0 e 2,0 m de altura do solo e em toda a extensão 
do perímetro da planta. $\mathrm{O}$ material foi encaminhado ao Laboratório de Qualidade da Fruta e Melhoramento do Centro de Citricultura Sylvio Moreira/IAC.

As determinações de altura e diâmetro do fruto foram feitas por leitura direta de cada amostra, com auxílio de uma escala graduada, em centímetros. A avaliação da cor dos frutos foi realizada em leitura no colorímetro Minolta CR 300. A massa total dos frutos foi obtida, de uma só vez, em uma balança marca Filizola com capacidade de $15 \mathrm{~kg}$, e sensibilidade de $5 \mathrm{~g}$. O rendimento em suco foi determinado após esmagamento do fruto na extratora Organização Internacional Centenário (OIC) modelo OTTO 1800 (filtro com diâmetro interno $=26,11 \mathrm{~mm}$; comprimento $=265 \mathrm{~mm}$; furos de diâmetro $=0,6 \mathrm{~mm}$; área de vazão $=20 \%$ ) e calculado através da relação massa do suco/massa do fruto e expresso em porcentagem (\%). O teor de sólidos solúveis foi determinado por leitura direta no refratômetro B\&S, modelo RFM 330 e expresso em ${ }^{\circ}$ Brix. Os dados foram corrigidos pela temperatura e pela acidez do suco. A acidez foi obtida por titulação de $25 \mathrm{~mL}$ de suco, com uma solução de hidróxido de sódio de normalidade 0,3125 e usando-se fenolftaleína como indicadora. Foi calculada a relação sólidos solúveis/acidez fazendo-se o valor da acidez igual a 1. A vitamina C (ácido ascórbico) foi determinada pela titulação de $50 \mathrm{~mL}$ de suco, seguindo a metodologia de Chen (1995). Também foi realizada a contagem do número de sementes de dez frutos, os quais foram coletados juntamente com o material para análise físico-química. Os frutos foram seccionados transversalmente com corte superficial da casca e torção em torno de seu eixo e em seguida, as sementes foram extraídas com auxílio de espátula e mensurado o número total de sementes por fruto.

Análise dos dados

Para análise dos dados foi realizada a análise de variância (ANOVA) e aplicado o teste de Scott-Knott usando o software ASSISTAT (Silva \& Azevedo, 2016).

\section{RESULTADOS E DISCUSSÃO}

\section{Desenvolvimento vegetativo}

Os genótipos apresentaram, aos 24 meses, altura média de 2,41 m, variando de $1,67 \mathrm{~m}$ a $2,75 \mathrm{~m}$, assim como o volume de copa médio foi de $6,31 \mathrm{~m}^{3}$ variando de $1,86 \mathrm{~m}^{3}$ a $10,31 \mathrm{~m}^{3}$ (Tabela 1). Variações no tamanho de plantas cítricas são comuns em genótipos, em plantas enxertadas em diferentes porta-enxertos e fase de desenvolvimento da copa. Stenzel et al. (2005) avaliando laranja Folha Murcha enxertada em limão Cravo, aos 96 meses, obtiveram altura de 3,6 m e volume de copa de 22,1 $\mathrm{m}^{3}$. Para tangerina Fremont enxertada em limão Cravo, aos 9 anos, foi determinado um volume de copa de 5,4 m³ (Núñez et al., 2007). Em limão Siciliano cultivado no estado do Ceará em diferentes porta-enxertos e espaçamento adensado, Sombra et al. (2017) apresentaram, aos 24 meses, altura variando de $1,67 \mathrm{~m}$ a $2,18 \mathrm{~m}$, corroborando com os dados deste trabalho, e volume de copa de $0,55 \mathrm{~m}^{3}$ a $4,34 \mathrm{~m}^{3}$ inferior aos encontrados nos genótipos aqui estudados. Na avaliação aos 14 meses não foram observadas diferenças significativas nas alturas entre os genótipos, mas em volume de copa houve diferença nos acessos Deba Ahmed e Allen Eureka, que apresentaram menores valores e Harvey que apresentou o maior volume (Tabela 1). Quando avaliados, aos 24 meses, Allen Eureka, Woglum e Genova se destacaram com as menores alturas, respectivamente $1,67 \mathrm{~m}, 1,97 \mathrm{~m}$ e $1,93 \mathrm{~m}$, diferindo do restante dos materiais. Em relação ao volume de copa, Harvey, Amber, Kusner, Meyer e Cook Eureka tiveram os maiores volumes aos dois anos de plantio, enquanto Allen Eureka e Genova com volumes de 1,86 $\mathrm{m}^{3} \mathrm{e}$ 3,29 $\mathrm{m}^{3}$ foram os menores (Tabela 1).

Desta forma, buscando genótipos de porte menor para adensamento de plantio, as copas Allen Eureka e Genova se mostram como potenciais, apresentando incremento de altura de planta entre as avaliações de $0,12 \mathrm{~m}$ e $0,13 \mathrm{~m}$ e incremento de volume de copa de $1,77 \mathrm{~m}^{3}$ e $1,99 \mathrm{~m}^{3}$ (Tabela 1).

\section{Características físico-químicas}

As características físicas dos frutos colhidos em maio de 2017 mostraram que os materiais avaliados apresentaram um número elevado de sementes, como é esperado para limões. O genótipo Harvey apresentou a menor média com 11 sementes fruto $^{-1}$, e apesar de terem diferenças estatísticas entre eles, segundo a classificação de Oliveira \& Scivittaro (2011), todos se enquadram nas classes de "cultivares com semente" (6 a 20 sementes fruto $^{-1}$ ) e "cultivares com muita semente" (mais de 21 sementes fruto $^{-1}$ ) (Tabela 2).

A massa dos frutos variou de 138,66 g a 396,33 g, sendo que Allen Eureka, Jaraguá do Sul e Woglum apresentaram os frutos com menores massas $(138,66 \mathrm{~g}, 140,66 \mathrm{~g}$ e $140,66 \mathrm{~g}$, respectivamente). Kusner e Meyer com médias de $219,00 \mathrm{~g}$ e 228,66 g foram os frutos de maior massa, com destaque para Deba Ahmed que se mostrou um genótipo 
exótico e com frutos extremamente grandes, com massa média de 396,33 g. (Tabela 2). Figueiredo et al. (2005) avaliando limão Eureka em diferentes porta-enxertos, encontrou para o porta-enxerto limão Cravo, massa média de $172 \mathrm{~g}(+/-26 \mathrm{~g})$, muito próximo aos resultados da maioria dos genótipos aqui estudados, os demais porta-enxertos variaram de $144 \mathrm{~g}(+/-16 \mathrm{~g})$ a $178 \mathrm{~g}(+/-19 \mathrm{~g})$.

Quanto ao tamanho do fruto, a altura variou de 7,06 cm até $11,16 \mathrm{~cm}$, diferindo Woglum, Genova, Allen Eureka, Jaraguá do Sul, Rigoni e Lisboa Bocaiuva com as menores

Tabela 1. Desenvolvimento vegetativo das copas de quatorze genótipos de limão. Cordeirópolis, SP (2016-2017)

\begin{tabular}{|c|c|c|c|c|c|c|c|c|c|}
\hline \multirow{2}{*}{ Genótipos } & \multicolumn{3}{|c|}{2016} & \multicolumn{3}{|c|}{2017} & \multicolumn{3}{|c|}{$\begin{array}{c}\text { Incremento de } 2016 \text { para } \\
2017\end{array}$} \\
\hline & $\begin{array}{l}\text { Altura } \\
(\mathrm{m})\end{array}$ & $\begin{array}{l}\text { Largura } \\
\text { (m) }\end{array}$ & $\begin{array}{l}\text { Volume de } \\
\text { Copa }\left(\mathbf{m}^{3}\right)\end{array}$ & $\begin{array}{l}\text { Altura } \\
(\mathrm{m})\end{array}$ & $\begin{array}{l}\text { Largura } \\
\text { (m) }\end{array}$ & $\begin{array}{l}\text { Volume de } \\
\text { Copa }\left(\mathbf{m}^{3}\right)\end{array}$ & $\begin{array}{c}\text { Altura } \\
(\mathrm{m})\end{array}$ & $\begin{array}{l}\text { Largura } \\
\text { (m) }\end{array}$ & $\begin{array}{l}\text { Volume de } \\
\text { Copa }\left(\mathbf{m}^{3}\right)\end{array}$ \\
\hline Jaraguá do Sul & $1,91 \mathrm{a}$ & $1,18 \mathrm{~b}$ & $1,41 \mathrm{~b}$ & $2,32 \mathrm{a}$ & $2,06 \mathrm{~b}$ & $5,20 \mathrm{~b}$ & $0,41 \mathrm{a}$ & $0,88 \mathrm{~b}$ & $3,78 \mathrm{c}$ \\
\hline Harvey & $1,86 \mathrm{a}$ & $2,51 \mathrm{c}$ & $6,19 \mathrm{c}$ & $2,51 \mathrm{a}$ & $2,52 \mathrm{a}$ & $8,97 \mathrm{a}$ & $0,65 \mathrm{a}$ & $0,01 \mathrm{~d}$ & $2,78 \mathrm{c}$ \\
\hline Allen Eureka & $1,55 \mathrm{a}$ & $0,91 \mathrm{a}$ & $0,69 \mathrm{a}$ & $1,67 \mathrm{~b}$ & $1,40 \mathrm{c}$ & $1,86 \mathrm{c}$ & $0,12 \mathrm{~b}$ & $0,49 \mathrm{c}$ & $1,17 \mathrm{c}$ \\
\hline Amber & $1,86 \mathrm{a}$ & $1,35 \mathrm{~b}$ & $1,82 \mathrm{~b}$ & $2,49 \mathrm{a}$ & $2,54 \mathrm{a}$ & $8,41 \mathrm{a}$ & $0,63 \mathrm{a}$ & $1,19 \mathrm{a}$ & $6,59 \mathrm{~b}$ \\
\hline São Matheus & $1,99 \mathrm{a}$ & $1,30 \mathrm{~b}$ & $1,77 \mathrm{~b}$ & $2,68 \mathrm{a}$ & $2,13 \mathrm{~b}$ & $6,62 \mathrm{~b}$ & $0,69 \mathrm{a}$ & $0,83 \mathrm{~b}$ & $4,85 \mathrm{c}$ \\
\hline Geórgia & $1,93 \mathrm{a}$ & $1,16 \mathrm{~b}$ & $1,39 \mathrm{~b}$ & $2,60 \mathrm{a}$ & $2,20 \mathrm{~b}$ & $6,56 \mathrm{~b}$ & $0,67 \mathrm{a}$ & $1,04 \mathrm{~b}$ & $5,17 \mathrm{~b}$ \\
\hline Kusner & $2,01 \mathrm{a}$ & $1,28 \mathrm{~b}$ & $1,77 \mathrm{~b}$ & $2,77 \mathrm{a}$ & $2,66 \mathrm{a}$ & $10,31 \mathrm{a}$ & $0,76 \mathrm{a}$ & $1,38 \mathrm{a}$ & $8,53 \mathrm{a}$ \\
\hline Lisboa & $1,84 \mathrm{a}$ & $1,30 \mathrm{~b}$ & $1,65 \mathrm{~b}$ & $2,33 \mathrm{a}$ & $2,03 \mathrm{~b}$ & $5,07 \mathrm{~b}$ & $0,49 \mathrm{a}$ & $0,73 \mathrm{c}$ & $3,41 \mathrm{c}$ \\
\hline Bocaiuva & & & & & & & & & \\
\hline Meyer & $2,06 \mathrm{a}$ & $1,45 \mathrm{~b}$ & $2,28 \mathrm{~b}$ & $2,54 \mathrm{a}$ & $2,46 \mathrm{a}$ & $8,14 \mathrm{a}$ & $0,48 \mathrm{a}$ & $1,01 \mathrm{~b}$ & $5,86 \mathrm{~b}$ \\
\hline Woglum & $1,81 \mathrm{a}$ & $1,20 \mathrm{~b}$ & $1,38 \mathrm{~b}$ & $1,96 \mathrm{~b}$ & $2,06 \mathrm{~b}$ & $4,38 \mathrm{~b}$ & $0,15 \mathrm{~b}$ & $0,86 \mathrm{~b}$ & $3,01 \mathrm{c}$ \\
\hline Cook Eureka & $2,08 \mathrm{a}$ & $1,19 \mathrm{~b}$ & $1,57 \mathrm{~b}$ & $2,75 \mathrm{a}$ & $2,54 \mathrm{a}$ & $9,35 \mathrm{a}$ & $0,67 \mathrm{a}$ & $1,35 \mathrm{a}$ & $7,77 \mathrm{a}$ \\
\hline Rigoni & $1,76 \mathrm{a}$ & $1,25 \mathrm{~b}$ & $1,51 \mathrm{~b}$ & $2,40 \mathrm{a}$ & $1,91 \mathrm{~b}$ & $4,65 \mathrm{~b}$ & $0,64 \mathrm{a}$ & $0,66 \mathrm{c}$ & $3,14 \mathrm{c}$ \\
\hline Genova & $1,80 \mathrm{a}$ & $1,17 \mathrm{~b}$ & $1,30 \mathrm{~b}$ & $1,93 \mathrm{~b}$ & $1,76 \mathrm{c}$ & $3,29 \mathrm{c}$ & $0,13 \mathrm{~b}$ & $0,59 \mathrm{c}$ & $1,99 \mathrm{c}$ \\
\hline Deba Ahmed & $1,96 \mathrm{a}$ & $0,95 \mathrm{a}$ & $0,92 \mathrm{a}$ & $2,69 \mathrm{a}$ & $1,95 \mathrm{~b}$ & $5,50 \mathrm{~b}$ & $0,73 \mathrm{a}$ & $1,00 \mathrm{~b}$ & $4,57 \mathrm{c}$ \\
\hline
\end{tabular}

Médias seguidas de mesma letra, na coluna, não diferem entre si pelo teste de Scott-Knott ao nível de 1\% de probabilidade.

Tabela 2. Características físicas dos frutos de quatorze genótipos de limão. Cordeirópolis, SP (2016-2017)

\begin{tabular}{lcccccc}
\hline \multicolumn{1}{c}{ Genótipos } & Semente $\left(\mathbf{n}^{\circ}\right)$ & Massa (g) & Altura (A) cm & Largura $(\mathbf{L}) \mathbf{c m}$ & $\mathbf{A} / \mathbf{L}$ & $\begin{array}{c}\text { Cor da } \\
\text { Casca* }\end{array}$ \\
\hline Jaraguá do Sul & $23,23 \mathrm{~b}$ & $140,66 \mathrm{a}$ & $7,36 \mathrm{a}$ & $6,16 \mathrm{a}$ & $1,19 \mathrm{~b}$ & $-5,97 \mathrm{c}$ \\
Harvey & $11,10 \mathrm{c}$ & $189,00 \mathrm{~b}$ & $8,30 \mathrm{~b}$ & $6,80 \mathrm{ab}$ & $1,22 \mathrm{~b}$ & $-4,95 \mathrm{c}$ \\
Allen Eureka & $21,10 \mathrm{~b}$ & $138,66 \mathrm{a}$ & $7,30 \mathrm{a}$ & $6,10 \mathrm{a}$ & $1,19 \mathrm{~b}$ & $-3,74 \mathrm{~b}$ \\
Amber & $19,00 \mathrm{~b}$ & $193,33 \mathrm{~b}$ & $8,30 \mathrm{~b}$ & $6,96 \mathrm{ab}$ & $1,19 \mathrm{~b}$ & $-5,80 \mathrm{c}$ \\
São Matheus & $21,53 \mathrm{~b}$ & $177,00 \mathrm{~b}$ & $7,90 \mathrm{~b}$ & $6,73 \mathrm{ab}$ & $1,17 \mathrm{~b}$ & $-3,30 \mathrm{~b}$ \\
Geórgia & $20,93 \mathrm{~b}$ & $174,66 \mathrm{~b}$ & $8,30 \mathrm{~b}$ & $6,43 \mathrm{ab}$ & $1,28 \mathrm{a}$ & $-5,42 \mathrm{c}$ \\
Kusner & $18,90 \mathrm{~b}$ & $219,00 \mathrm{c}$ & $8,80 \mathrm{~b}$ & $7,16 \mathrm{ab}$ & $1,23 \mathrm{~b}$ & $-5,40 \mathrm{c}$ \\
Lisboa Bocaiuva & $34,20 \mathrm{a}$ & $172,33 \mathrm{~b}$ & $7,60 \mathrm{a}$ & $6,76 \mathrm{ab}$ & $1,12 \mathrm{c}$ & $0,43 \mathrm{a}$ \\
Meyer & $28,50 \mathrm{a}$ & $228,66 \mathrm{c}$ & $8,13 \mathrm{~b}$ & $7,40 \mathrm{ab}$ & $1,10 \mathrm{c}$ & $-3,21 \mathrm{~b}$ \\
Woglum & $24,76 \mathrm{~b}$ & $140,66 \mathrm{a}$ & $7,06 \mathrm{a}$ & $6,40 \mathrm{ab}$ & $1,10 \mathrm{c}$ & $-3,22 \mathrm{~b}$ \\
Cook Eureka & $21,73 \mathrm{~b}$ & $168,66 \mathrm{~b}$ & $8,00 \mathrm{~b}$ & $6,53 \mathrm{ab}$ & $1,22 \mathrm{~b}$ & $-6,66 \mathrm{c}$ \\
Rigoni & $21,46 \mathrm{~b}$ & $165,66 \mathrm{~b}$ & $7,43 \mathrm{a}$ & $6,73 \mathrm{ab}$ & $1,10 \mathrm{c}$ & $-1,54 \mathrm{a}$ \\
Genova & $34,56 \mathrm{a}$ & $185,66 \mathrm{~b}$ & $7,13 \mathrm{a}$ & $7,16 \mathrm{ab}$ & $0,99 \mathrm{~d}$ & $-2,29 \mathrm{~b}$ \\
Deba Ahmed & $32,50 \mathrm{a}$ & $396,33 \mathrm{~d}$ & $11,16 \mathrm{c}$ & $8,70 \mathrm{~b}$ & $1,28 \mathrm{a}$ & $-4,32 \mathrm{c}$ \\
\hline
\end{tabular}

Médias seguidas de mesma letra, na coluna, não diferem entre si pelo teste de Scott-Knott ao nível de 1\% de probabilidade.

*Os dados da Cor de Casca foram transformados $(\mathrm{X}=\mathrm{X}+\mathrm{C}$, sendo $\mathrm{C}=8)$ para análise estatística. 
alturas. A largura variou de $6,10 \mathrm{~cm}$ a $7,40 \mathrm{~cm}$ não diferindo estatisticamente os materiais, exceto Deba Ahmed com altura de $8,70 \mathrm{~cm}$. Quanto à relação altura $\mathrm{x}$ largura $(\mathrm{A} / \mathrm{L})$, que indica o formato alongado ou arredondado do fruto, o limão Genova diferiu dos demais apresentando a menor relação $(0,99)$, enquanto Deba Ahmed e Geórgia apresentaram a maior relação $(1,28)$ (Tabela 2). Almeida (2014) trabalhando com limão Siciliano enxertado em limão Cravo no Ceará, obteve para colheita aos 77 dias, altura de fruto de $6,01 \mathrm{~cm}$, largura $5,18 \mathrm{~cm}$ e a relação entre eles de 1,16 , valores inferiores aos materiais aqui avaliados.

Com relação às características químicas dos frutos, foram observadas diferenças significativas no rendimento em suco para Deba Ahmed, Jaraguá do Sul, Harvey e Kusner, que foram os genótipos com menores rendimentos, variando de $37,70 \%$ a 40,20\%. Os demais materiais não diferiram estatisticamente entre si e variaram de $45,43 \%$ a 58,23\%, esse último rendimento alcançado pelo Meyer (Tabela 3). Para citros, nas condições brasileiras, Santos Filho et al. (2005) consideram como rendimento em suco adequado um mínimo de $35 \%$ a $45 \%$, variando em função do cultivar. Sharma et al. (2006) encontraram valores de $52,3 \%, 50,2 \%$ e 53,8\% para os genótipos Eureka, Lisbon e Kagzi Kalan, respectivamente, muito próximos dos cultivares deste trabalho. No estado do Ceará, obteve-se para limão Siciliano, rendimento em suco de $42,78 \%$ no $91^{\circ}$ dia após a queda de pétalas (Almeida, 2014), enquanto em São Paulo, Figueiredo et al. (2005), com limão Eureka $\mathrm{km} 47$ enxertado em diversos porta-enxertos, alcançaram valores que variaram de $47 \%$ a 53\%, mostrando que além do genótipo copa, o material do porta-enxerto influencia na qualidade dos frutos. É importante na hora da escolha do material copa/porta-enxerto, além dos aspectos fitotécnicos e fitossanitários, considerar a qualidade do fruto gerado.

A quantidade de vitamina $\mathrm{C}$ variou de 23,77 a 43,94 mg $100 \mathrm{~mL}^{-1}$ entre os genótipos avaliados. Os limões Amber, Meyer e Cook Eureka apresentaram os maiores valores, enquanto os menores teores foram observados em Allen Eureka, Woglum e Rigoni (Tabela 3). Yekeler et al. (2013) avaliando Siciliano obteve teores de vitamina $\mathrm{C}$ de $24,8 \mathrm{mg} 100 \mathrm{~mL}^{-1}$.

Para o índice tecnológico destacou-se o limão Meyer com $1,98 \mathrm{~kg}$ caixa $^{-1}$ de $40,8 \mathrm{~kg}$, enquanto os outros materiais se diferiram em dois grupos: o primeiro variou de 1,50 a 1,61 kg caixa ${ }^{-1}$, e o segundo, com os menores rendimentos, ficou entre 1,13 e 1,44 $\mathrm{kg}$ caixa $^{-1}$. Quatro genótipos (Woglum, Rigoni, Allen Eureka e Deba Ahmed) apresentaram os menores valores de sólidos solúvies (entre 6,83 e 7,40 ${ }^{\circ}$ Brix). Amber, Meyer e Cook Eureka apresentaram os melhores valores de sólidos solúveis (SS), ao redor de 8,0 ${ }^{\circ}$ Brix (Tabela 3). González-Molina et al. (2008) estudando diversos genótipos de limão obtiveram valores de 8,2 a $9,8^{\circ}$ Brix, e para limão Siciliano, Jacomino et al. (2003) encontraram valores entre 6,9 a 7,3 ${ }^{\circ}$ Brix. Em estudos no estado de São

Tabela 3. Características químicas dos frutos de quatorze genótipos de limão. Cordeirópolis, SP (2016-2017)

\begin{tabular}{lcccccc}
\hline \multicolumn{1}{c}{ Genótipos } & $\begin{array}{c}\text { Vitam. C } \\
\left(\mathbf{m g ~ 1 0 0 ~} \mathbf{~ m L}^{-1}\right)\end{array}$ & $\begin{array}{c}\text { Rd. Suco \% } \\
(\mathbf{m} / \mathbf{m})\end{array}$ & $\begin{array}{c}\text { Acidez } \mathbf{~} \\
\mathbf{1 0 0} \mathbf{~ m L}^{-1}\end{array}$ & ${ }^{\circ}$ Brix corr. & Ratio & $\begin{array}{c}\text { SST kg } \\
\text { caixa }^{-1}\end{array}$ \\
\hline Jaraguá do Sul & $38,17 \mathrm{a}$ & $39,93 \mathrm{~b}$ & $4,90 \mathrm{a}$ & $7,86 \mathrm{a}$ & $1,60 \mathrm{a}$ & $1,28 \mathrm{c}$ \\
Harvey & $37,97 \mathrm{a}$ & $39,16 \mathrm{~b}$ & $5,00 \mathrm{a}$ & $7,73 \mathrm{a}$ & $1,53 \mathrm{a}$ & $1,23 \mathrm{c}$ \\
Allen Eureka & $23,77 \mathrm{~b}$ & $47,40 \mathrm{a}$ & $4,84 \mathrm{a}$ & $7,06 \mathrm{~b}$ & $1,46 \mathrm{a}$ & $1,36 \mathrm{c}$ \\
Amber & $43,94 \mathrm{a}$ & $46,06 \mathrm{a}$ & $4,98 \mathrm{a}$ & $8,06 \mathrm{a}$ & $1,60 \mathrm{a}$ & $1,50 \mathrm{~b}$ \\
São Matheus & $35,05 \mathrm{a}$ & $49,03 \mathrm{a}$ & $5,11 \mathrm{a}$ & $7,96 \mathrm{a}$ & $1,56 \mathrm{a}$ & $1,58 \mathrm{~b}$ \\
Geórgia & $38,20 \mathrm{a}$ & $45,43 \mathrm{a}$ & $5,07 \mathrm{a}$ & $7,80 \mathrm{a}$ & $1,53 \mathrm{a}$ & $1,44 \mathrm{c}$ \\
Kusner & $37,57 \mathrm{a}$ & $40,20 \mathrm{~b}$ & $5,44 \mathrm{a}$ & $7,93 \mathrm{a}$ & $1,46 \mathrm{a}$ & $1,30 \mathrm{c}$ \\
Lisboa Bocaiuva & $28,01 \mathrm{~b}$ & $49,83 \mathrm{a}$ & $4,67 \mathrm{~b}$ & $7,53 \mathrm{a}$ & $1,60 \mathrm{a}$ & $1,54 \mathrm{~b}$ \\
Meyer & $39,17 \mathrm{a}$ & $58,23 \mathrm{a}$ & $4,31 \mathrm{~b}$ & $8,36 \mathrm{a}$ & $1,93 \mathrm{a}$ & $1,98 \mathrm{a}$ \\
Woglum & $26,84 \mathrm{~b}$ & $47,96 \mathrm{a}$ & $4,47 \mathrm{~b}$ & $6,83 \mathrm{~b}$ & $1,50 \mathrm{a}$ & $1,33 \mathrm{c}$ \\
Cook Eureka & $39,62 \mathrm{a}$ & $46,80 \mathrm{a}$ & $5,37 \mathrm{a}$ & $8,26 \mathrm{a}$ & $1,53 \mathrm{a}$ & $1,58 \mathrm{~b}$ \\
Rigoni & $25,84 \mathrm{~b}$ & $49,80 \mathrm{a}$ & $4,16 \mathrm{~b}$ & $6,96 \mathrm{~b}$ & $1,66 \mathrm{a}$ & $1,41 \mathrm{c}$ \\
Genova & $32,61 \mathrm{~b}$ & $52,10 \mathrm{a}$ & $5,18 \mathrm{a}$ & $7,60 \mathrm{a}$ & $1,46 \mathrm{a}$ & $1,61 \mathrm{~b}$ \\
Deba Ahmed & $29,59 \mathrm{~b}$ & $37,70 \mathrm{~b}$ & $4,90 \mathrm{a}$ & $7,40 \mathrm{~b}$ & $1,53 \mathrm{a}$ & $1,13 \mathrm{c}$ \\
\hline
\end{tabular}

Médias seguidas de mesma letra, na coluna, não diferem entre si pelo teste de Scott-Knott ao nível de $1 \%$ de probabilidade. Vitam. = Vitamina, Rd. $=$ Rendimento, corr. $=$ Corrigido, SST $=$ Sólidos Solúveis Totais. 
Paulo com Eureka km 47 enxertado sobre limão Cravo, o teor de SS alcançado foi de 9,3 ${ }^{\circ}$ Brix (Figueiredo et al., 2005). Esses números mostram que o teor de sólidos solúveis é variável com o genótipo da copa, porta-enxerto e condições ambientais.

A acidez, importante parâmetro para estudos com limão, variou de 4,16 a 5,44 g $100 \mathrm{~mL}^{-1}$, sendo que os genótipos se dividiram em dois grupos: um com menor acidez, de 4,16 g $100 \mathrm{~mL}^{-1}$ (Rigoni) a 4,67 g $100 \mathrm{~mL}^{-1}$

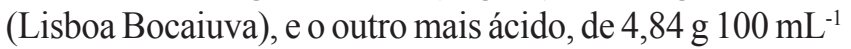
(Allen Eureka) a 5,44 g $100 \mathrm{~mL}^{-1}$ (Kusner) (Tabela 3). Nascimento et al. (2005) em estudo com laranjas encontrou para as variedades Pêra, Charmute de Brotas, Valência e Folha Murcha, acidez de 0,89, 0,92, 1,32 e 1,10 g $100 \mathrm{~mL}^{-1}$ respectivamente, que quando comparado com os dados de limões mostra a grande diversidade de acidez entre as frutas cítricas. Silva et al. (2006) estudando lima ácida

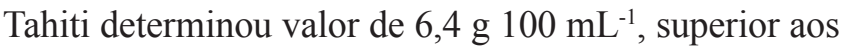
dados aqui encontrados. Em limão Siciliano aos 77 dias após a floração, a acidez encontrada foi de $5,71 \mathrm{~g} 100 \mathrm{~mL}^{-1}$ corroborando com os encontrados nos genótipos aqui estudados (Almeida, 2014).

O ratio, que é a relação entre o teor de sólidos solúveis e a acidez, ficou entre 1,46 e 1,93, e não houve diferença significativa para essa relação entre os materiais. No entanto, o genótipo Meyer teve o maior ratio $(1,93)$, visto que também teve alto teor de SS. Almeida (2014) apresenta valor de ratio muito próximo $(1,57)$ dos aqui encontrados para limão Siciliano nas condições do nordeste brasileiro.

É importante ressaltar que novos estudos são necessários para avaliação do comportamento desses materiais quanto a pragas e doenças, porta-enxertos, níveis de adubação e clima.

\section{CONCLUSÕES}

Dos quatorze genótipos estudados, os genótipos Allen Eureka e Genova se destacaram pelo menor desenvolvimento de copa. Quanto à qualidade dos frutos no ponto de maturação, Meyer e Genova apresentaram os melhores rendimento e teores dos aspectos avaliados.

\section{REFERÊNCIAS}

Almeida, B. A. (2014). Determinação do estádio ótimo de maturação a colheita do limão 'Siciliano', produzidos no estado do Ceará (Dissertação de mestrado). Fortaleza: Universidade Federal do Ceará.
Alves, F. L. (1986). Características fisicas, químicas e físico-químicas de frutas de cultivares de limão Citrus limom (L.) Burm (Dissertação de mestrado). Botucatu: UNESP.

Amaro, A. A., \& Maia, M. L. (1997). Produção e comércio de laranja e suco no Brasil. Informações Econômicas, 27(7), 11-27.

Amaro, A. A., Caser, D. V., \& Negri, J. D. D. (2003). Tendências na produção e comércio de limão. Informações Econômicas, 33(4), 37-47.

Carvalho, V. D., \& Nogueira, D. J. P. (1979). Qualidade, maturação e colheita dos citros. Informe Agropecuário, $5(52), 62-67$.

Chen, H. Z. (1995). Determination of ascorbic acid in drinks using cyanide-selective electrode with the addition of iodine. Huaxue Chuanganqi, 15(4), 295-297.

Curk, F., Ollitrault, F., Garcia-Lor, A., Luro, F., Navarro, L., \& Ollitrault, P. (2016). Phylogenetic origin of limes and lemons revealed by cytoplasmic and nuclear markers. Annals of Botany, 117(4), 565-583.

Donadio, L. C., \& Stuchi, E. S. (2001). Adensamento de plantio e ananicamento de citrus (70 pp.). Jaboticabal: UNESP.

Figueiredo, J. O., Negri, J. D., Mattos Júnior, D. M., Pio, R. M., Laranjeira, F. F., \& Garcia, V. X. P. (2005). Comportamento de quatorze porta-enxertos para limão Eureka km 47 na região de Araraquara-SP. Revista Brasileira de Fruticultura, 27(1), 73-76.

Food and Agriculture Organization - FAO. (2016). Faostat: production. Recuperado em: 20 de março 2018, de http://faostat.fao.org/

González-Molina, E., Moreno, D. A., \& García-Viguera, C. (2008). Genotype and harvest time influence the phytochemical quality of fino lemon juice (Citrus limon (L.) Burm. F.). Journal of Agricultural and Food Chemistry, 56(5), 1669-1675.

Jacomino, A. P., Mendonça, K., \& Kluge, R. A. (2003). Armazenamento refrigerado de limões 'Siciliano' tratados com etileno. Revista Brasileira de Fruticultura, 25(1), 45-48.

Landa, R. I. (2014). Análisis de la producción y comercialización del limón en Argentina (Trabajo final). Facultad de Ciencias Agrarias, Universidad Católica Argentina, Buenos Aires. Recuperado em: 20 de março 
2018, http://bibliotecadigital.uca.edu.ar/repositório/tesis/ analisis-produccionco-mercializacion-limon.pdf

Mendel, K. (1956). Rootstock-scion relationships in Shamouti trees on light soil. Ktavim, Rehovot, 6, 35-60.

Nascimento, L. M., Pompeu Júnior, J., Negri, J. D., Zara, F. A., \& Chignolli, R. C. (2005). Laranja Charmute de Brotas: promissora variedade tardia. Laranja, 26(1), 69-75.

Núñez, E. E., Mourão Filho, F.A. A., \& Stuchi, E. S. (2007). Desenvolvimento vegetativo, produção e qualidade de frutos da tangerina 'Fremont' sobre quatro porta-enxertos. Revista Brasileira de Fruticultura, 29(2), 308-312.

Oliveira, R. P., \& Scivittaro, W. B. (2011). Cultivo de citros sem semente (377 pp.). Pelotas: EMBRAPA.

Pio, R. M. (1992). Caracterização e avaliação de frutos de oito variedades do grupo das tangerinas (Dissertação de mestrado). Piracicaba: ESALQ.

Pio, R. M., Figueiredo, J. O., Stuchi, E. S., \& Cardoso, S. A. B. (2005). Variedades Copa. In D. Mattos Junior, J. D. Negri, R. M. Pio, \& J. Pompeu Junior (Eds.), Citros (cap. 3, pp. 37-60). Campinas: Instituto Agronômico, Fundag.

Santos Filho, H. P., Magalhães, A. F. J., \& Coelho, Y. S. (2005). Citros: o produtor pergunta, a Embrapa responde (219 pp.). Brasília: Embrapa Informações Tecnológicas.

Sharma, R. R., Singh, R., \& Saxena, S. K. (2006). Characteristics of citrus fruits in relation to granulation. Scientia Horticulturae, 111(1), 91-96.

Silva, C. R., Jordão, F., Alves Júnior, J., Silva, T. J. A., Folegatti, M. V., \& Maschio, R. (2006). Respostas de Limeira Ácida Tahiti à suspensão da irrigação em diferentes períodos fenológicos. Laranja, 27(2), 321-339.
Silva, F. A. S., \& Azevedo, C. A. V. (2016). The Assistat Software Version 7.7 and its use in the analysis of experimental data. African Journal of Agricultural Research, 11(39), 3733-3740.

Sombra, K. E. S., Loureiro, F. L. C., Costa e Silva, A. C., Passos, O. S., \& Bastos, D. C. (2017) Desenvolvimento inicial de limoeiro siciliano sob diferentes porta-enxertos em espaçamento adensado no semiárido do Ceará. In A. T. S. Alfaro, D. G. Trojan (Org.), Descobertas das ciências agrárias e ambientais (cap. 3, pp. 164-171). Ponta Grossa: Atena.

Stenzel, N. M. C., Neves, C. S. V. J., Gonzalez, M. G. N., Scholz, M. B. S., \& Gomes, J. C. (2005). Desenvolvimento vegetativo, produção e qualidade dos frutos da laranjeira 'Folha Murcha' sobre seis porta-enxertos no norte do Paraná. Ciência Rural, 35(6), 1281-1286.

Stuchi, E. S., \& Girardi, E. A. (2011). Adensamento de plantio deve ser o quarto elemento no manejo do HLB. Citricultura Atual, 14(81), 12-16.

Yekeler, F. Z., Ozyurek, H., \& Tamer, C. E. (2013). A functional beverage: lemonade. International Journal of Agricultural, Biosystems Science and Engineering, 7(7), 617-620.

Recebido: Outubro 24, 2017 Aceito: Outubro 09, 2018

Como citar: Ferreira, R. V., Bastianel, M., Azevedo, F. A., \& De Negri, J. D. (2018). Desenvolvimento vegetativo e características físico-químicas dos frutos de quatorze genótipos de limão. Citrus Research \& Technology, 39, e1037. https://doi.org/10.4322/crt.14818. 
